\title{
Análisis de la política fiscal descrita en la constitución vigente del Ecuador
}

\author{
A fiscal policy analysis described from current Ecuadorian constitution
}

Lenín Agustín Chamba Bastidas. ${ }^{1}$, Adriana Margarita Morales Noriega. ${ }^{2}$, Henry David Vásconez Vásconez . ${ }^{3}$ \& Daniel Antonio Cabrera Bravo. ${ }^{4}$

\section{https://doi.org/10.33262/visionariodigital.v4i1.1106}

\begin{abstract}
.
The Ecuadorian economy in a large extent depends from the Fiscal Policy, since the execution of the Monetary Policy is limited, because the country in 2000 adopted a dollarization after a strong and pronounced financial crisis; which was responsible for the increase in poverty levels and limited level of growth and development. Ecuador also keeps a fiscal deficit close to 3.300 million dollars, but this is not the unique problem that the country has, since in order to counteract the gap, public debt keeps growing and it is no healthy for Ecuadorian economy if the state do not manage the tax collection system, public debt and the state spending to achieve an economic and development growth. It is not an easy task for the Ecuadorian authorities to enforce the Fiscal Policy objectives ordered in current Constitution, as it have proved insufficient to solve the competitiveness and productive structural problem that Ecuador has. It is necessary to describe the Fiscal Policy established in the country, in order to assess whether the government is properly managing state goods and services, since its actions are based on favorable conditions generation for the entire population development.
\end{abstract}

\footnotetext{
${ }^{1}$ Escuela Superior Politécnica de Chimborazo, Chimborazo, Riobamba, lenin.chamba@espoch.edu.ec

${ }^{2}$ Escuela Superior Politécnica de Chimborazo, Chimborazo, Riobamba, adriana.morales@espoch.edu.ec

${ }^{3}$ Universidad Nacional de Chimborazo, Chimborazo, Riobamba, hdvasconezv@ hotmail.com

${ }^{4}$ Escuela Superior Politécnica de Chimborazo, Chimborazo, Riobamba, alimentamx@gmail.com
} 
Keywords: Fiscal Policy, Economic growth, Economic development, Ecuadorian constitution.

\section{Resumen.}

La Economía de Ecuador depende en gran medida de la Política Fiscal, ya que la ejecución de la Política Monetaria es muy limitada por el proceso de dolarización que optó el Ecuador a partir del año 2000 tras una muy pronunciada y fuerte crisis financiera; la cual fue la responsable del incremento de los niveles de pobreza y limitados niveles de crecimiento desarrollo. Por otro lado, el país mantiene un déficit fiscal cercano a 3.300 millones de dólares, problema que no es el único, ya que, en aras de contrarrestar dicha brecha, en el Ecuador sigue creciendo el endeudamiento, lo cual no es saludable mientras no se gestione la deuda pública, el gasto estatal y los sistemas de recaudación tributaria para impulsar el crecimiento y desarrollo económico. No es tarea fácil para las autoridades ecuatorianas el cumplimiento de los objetivos de la Política Fiscal ordenados en la Constitución vigente, ya que han resultado no ser suficientes para dar solución al problema estructural de producción y competitividad que tiene el Ecuador. Es necesario describir la Política Fiscal instaurada en el país, con la finalidad de valorar si el gobierno está administrando adecuadamente los bienes y servicios estatales, ya que su accionar se basa en la generación de condiciones favorables para el desarrollo de toda la población.

Palabras claves: Política Fiscal, Crecimiento económico, Desarrollo económico, Constitución ecuatoriana

\section{Introducción.}

A partir de enero del año 2000, la política fiscal se convirtió en el único instrumento que el gobierno tendría para intervenir en el ámbito económico; ya que, al adoptar el dólar como moneda del sistema económico ecuatoriano, invalidó el accionar de la política monetaria y cambiaria debido a la imposibilidad de controlar el tipo de cambio y el flujo de la moneda; desde entonces la situación fiscal del Ecuador, se ha convertido en uno de los problemas principales que los gobiernos han tenido que enfrentar. (Carrillo, 2017). Específicamente en la economía ecuatoriana ha existido niveles de déficit fiscal altos y no habido una adecuada generación de ingresos, por lo cual el estado ha tenido que endeudarse para poder resolver la brecha económica existente y así lograr que la población ecuatoriana pueda satisfacer sus necesidades.

Ante la necesidad de fortalecer la política fiscal en la economía ecuatoriana, la Asamblea Nacional en el año 2007, redacta la Nueva Constitución del Ecuador, la cual fue aprobada por la mayoría de la población en el 2008, esta normativa constitucional a través del establecimiento de un sistema económico social y solidario, considera al ser humano como 
sujete y fin del accionar económico. A través de esta nueva normativa constitucional se incorpora a la progresividad, la equidad, la suficiencia recaudatoria, la priorización de impuestos directos y la responsabilidad ambiental como pilares fundamentales que garantizan una sociedad más justa y alineada a los parámetros del Buen Vivir. (Servicio de Rentas Internas, 2012).

La presente investigación tiene como objetivo analizar los objetivos de política fiscal descritos en la Constitución del Ecuador. Para este análisis es necesario observar la información obtenida en el período del expresidente Economista Rafael Correa y del actual presidente, el Licenciado Lenin Moreno. El análisis se ha realizado en base al ámbito del derecho y la economía, ya que existe una relación muy cercana entre estas dos ciencias.

\section{Revisión de literatura}

\section{Política Fiscal}

La política fiscal es aquella que se encarga de analizar las tasas efectivas de los impuestos y el gasto público del gobierno central, con la finalidad de modificar la demanda agregada y así mantener una ocupación plena y precios estables, en otras palabras, el propósito de la política fiscal es alcanzar una estabilización en la economía. (Otalora, 2009). La política fiscal es también la encargada del establecimiento de impuestos y gastos públicos, con el objetivo de contribuir al amortiguamiento de las oscilaciones del ciclo económico y contribuir de esta manera a mantener una economía en crecimiento, donde la tasa de empleo sea elevada y no existan niveles volátiles de inflación. (Samuelson \& Nordhaus, 2014). Esta política es también el conjunto de políticas sobre ingreso y gasto que determinan el monto de los recursos disponibles y los fines en los que se invierten para el desarrollo de las actividades estatales. Esta política es uno de los instrumentos esenciales del Estado para redistribuir la riqueza, corregir fallas en el mercado, garantizar los derechos humanos y avanzar progresivamente en la erradicación de la pobreza y la disminución de las desigualdades. (Center for Economic and Social Rights, 2017)

La política fiscal, entonces se la puede definir como las medidas que adopta el gobierno central para poder recaudar ingresos a través de la imposición de tributos a la población, con la finalidad de cubrir los gastos que se generan en el sector público y así contribuir a la satisfacción de las necesidades de la población, lo cual permita un adecuado equilibrio económico. En otras palabras y de acuerdo a lo establecido en la constitución vigente, es el Estado ecuatoriano el responsable de plantear una política fiscal adecuada.

\section{Tipos de Política Fiscal}

Las medidas económicas que establece un Gobierno buscan afectar directamente a la demanda agregada, de aquí se derivan dos tipos de política fiscal:

\section{Política Fiscal Expansiva}


Este tipo de política se aplica cuando existe una crisis económica y la demanda agregada resulta ser insuficiente, ya que existe desempleo y también el potencial de producción no está siendo utilizado. Se utiliza esta política para incrementar los índices de empleo y crecimiento económico y los mecanismos de acción son:

- Aumento de gasto público. - se realiza esta medida para incrementar la producción y el empleo y así aumentar la demanda agregada.

- Disminución de impuestos. - se reducen las tasas impositivas con la finalidad de aumentar la renta de las familias y así puedan consumir. Por otro lado, se busca una disminución en los costos de las empresas para que puedan incrementar sus volúmenes de inversión.

\section{Política Fiscal Restrictiva}

Estas medidas se aplican cuando la economía presenta niveles excesivos de expansión y es necesario poner un límite para evitar que exista un aumento de los precios, es decir para que haya inflación. Los mecanismos de acción son:

- Reducción del gasto público. - el estado debe procurar disminuir las inversiones públicas, transferencias y compras para contraer la demanda y también el nivel de precios.

- Alza de impuestos. - con esta medida el nivel de renta de las familias disminuye con la finalidad de reducir el consumo y así las empresas se enfrentan a mayores costos lo cual disminuirá la inversión.

De acuerdo con el escenario económico actual, el Ecuador ha instaurado una política fiscal de tipo expansiva, la cual busca un aumento del gasto público, que a criterio del régimen busca una infraestructura productiva en el país y también la generación de empleos estables, sin embargo, se visualiza como este gasto está siendo financiado por deuda, lo cual está limitando el crecimiento de la economía ecuatoriana.

Analizando a la economía en el contexto fiscal en los últimos años, se pueden destacar los siguientes puntos: en 1999, la economía ecuatoriana atravesó shocks externos que afectaron drásticamente al país, por un lado, estuvo la pérdida del sucre como moneda oficial del país, el sistema financiero ecuatoriano estuvo a punto de quebrar, la actividad productiva también se vio afectada. Frente a estos acontecimientos, la economía necesitaba una urgente reactivación del aparato productivo. Posteriormente en el 2000, tras un incremento en el nivel de inversión y consumo, el Producto Interno Bruto creció en un 4\%, la inflación anual de su lado, cerro en $38 \%$ en el año 2001. Para el siguiente período el crecimiento del PIB fue del $4 \%$, como resultado de un aumento en el consumo e inversión; la inflación anual decreció notablemente, así de 96,10 \% en el 2000 se redujo al 37,7 \% en el año 2001. Sin embargo, a pesar de los intentos que se realizaron por fortalecer la economía ecuatoriana, el gasto 
corriente se incrementó de manera desacelerada y el gasto de capital aumentó de manera leve. (Álvarez, 2006).

Debido a la escasa actividad petrolera que hubo durante el año 2003, la economía sufrió un decrecimiento de un $2.7 \%$, pero también a partir de este año hubo un crecimiento importante en el pago de salarios al sector público. Posteriormente para el período 2005-2008, la política fiscal presentó cifras distintas a las alcanzadas anteriormente, ya que se registró un crecimiento promedio de los ingresos tributarios de casi el $11 \%$, lo cual al gobierno le permitió contar con un disponible para cubrir los gastos corrientes. Sin embargo, debido a la crisis inmobiliaria que sufrió Estados Unidos en el 2009, la economía a nivel mundial sufrió desequilibrios, Ecuador no fue la excepción ya que únicamente alcanzó una tasa de crecimiento de $0,58 \%$, siendo éste un nivel inferior respecto al alcanzado en el 2008 , el cual fue del 6,5\%. (Rozas, 2010)

Desde el año 2010, el nivel de gasto público empieza a crecer de una manera desacelerada,35\% para este año, ya que una serie de secretarias, subsecretarias y ministerios en nuestro país se crearon, entonces había que cancelar los haberes de los funcionarios públicos que ingresaban a trabajar en estas entidades. Se empezaba ya avizorar desajustes en la economía local, ya que a pesar de que la recaudación tributaria alcanzo los niveles más altos de la historia, el gobierno aumentó de igual manera el gasto público y generalmente lo utilizaba para cubrir aquellos gastos corrientes que se derivaban de su accionar político. Estas acciones significaron un déficit para el Sector Público no Financiero, el cual alcanzó el 5,3\% en el año 2014.

En nuestro país existe hoy una crisis fiscal de gran magnitud, hay un excesivo gasto público desde la bonanza petrolera, el país tiene que cubrir una brecha económica de 5.000 millones, la cual en gran medida de podría solucionar si existe una austeridad en el gasto público, si existe una generación de ingresos, focalización de subsidios, re perfilar el endeudamiento externo. El Ecuador necesita corregir la crisis fiscal, es decir, pagar las cuentas, no es momento de pagar bonos ni subsidiar a la economía; ya que según el Center for Economic and Social Rights, la Política Fiscal es uno de los instrumentos para erradicar la pobreza y actualmente la pobreza se ha convertido en el principal enemigo a nivel nacional y mundial.

La política fiscal en el Ecuador, aún no ha demostrado alcanzar los objetivos planteados en la normativa ecuatoriana vigente, ya que la tasa de crecimiento del Producto Interno Bruto en el año 2018 con relación al 2017, presentó una caída del 50\% aproximadamente, la deuda pública ha continuado creciendo y representa el 51\% del PIB aproximadamente y la pobreza se presenta en un $25 \%$ en los últimos años. Ante estos datos económicos bastante desproporcionales, es importante analizar la normativa de Ecuador y valorar si efectivamente el gobierno está cumpliendo con lo que determina la Ley. 
Según el artículo 1 del Código Civil vigente en el Ecuador, la Ley es una declaración de la voluntad soberana que, manifestada en la forma prescrita por la Constitución, manda, prohíbe o permite, lo que significa que el contenido de la ley es de cumplimiento obligatorio en el orden del artículo 425 de la carta magna, mismo que hace referencia a la jerarquía.

Considerando lo descrito en el párrafo que antecede, el Estado ecuatoriano a través de su dependencia correspondiente, debería cumplir con los objetivos descritos en la Constitución vigente artículo 245 respecto de la Política Fiscal:

La política fiscal tendrá como objetivos específicos: 1. El financiamiento de servicios, inversión y bienes públicos. 2. La redistribución del ingreso por medio de transferencias, tributos y subsidios adecuados. 3. La generación de incentivos para la inversión en los diferentes sectores de la economía y para la producción de bienes y servicios, socialmente deseables y ambientalmente aceptables. (Asamblea Nacional, 2008)

\section{Metodología.}

La investigación desarrollada es de tipo descriptiva, debido a que se analizará lo suscitado en la economía ecuatoriana y se los interpretará en el contexto actual de la política fiscal. En esta investigación se ha examinado registros, análisis e interpretaciones de las variables analizadas respecto a la economía del país, para de esta manera conocer el contexto económico en el aspecto fiscal. En cuanto al tratamiento de variables se realizó un análisis univariado, es decir se estudió una a una las variables con la finalidad de encontrar el impacto del accionar fiscal en la estabilidad económica del Ecuador.

Se analizó la política fiscal descrita en la Constitución a partir de algunas variables relacionadas a los objetivos ordenados en el artículo 285 de la ley suprema, sin embargo, estos objetivos establecidos en este artículo no evidencian una metodología definida de seguimiento, medición y/o valoración a efectos de conocer si aquellos se cumplieron o no, motivo por el cual se intentó describir las variables que a criterio de los autores se ha considerado pueden responder dichos objetivos.

\section{Resultados.}

La política fiscal tiene un rol fundamental en la recaudación y administración de recursos para la redistribución y disminución de las desigualdades sociales y económicas en una sociedad, por lo tanto, su impacto debe analizarse con indicadores que mayor explicación de al ejercicio de dicha política.

De acuerdo con lo establecido en el accionar fiscal, esta política persigue objetivos como: aceleración del crecimiento económico, ocupación total de los recursos productivos disponibles en el país para contribuir al desarrollo de un empleo estable y la estabilidad de los precios. Sin embargo, los objetivos que plantea la Constitución del Ecuador en cuanto a 
la política fiscal no son exactamente los mismos, pues evidencian el direccionamiento de este accionar en torno a la redistribución de los ingresos a la población, así como también la generación de ingresos para el desarrollo de las actividades productivas.

Debido a que en el país no se ha establecido una metodología adecuada para realizar una medición de los objetivos planteados en la política fiscal, se han definido variables que de alguna manera permitan demostrar lo actuado en cuanto a los objetivos planteados; entre esas variables macroeconómicas que se analizará, se encuentran las siguientes:

- PIB

- Deuda

- Pobreza

\section{PIB:}

Una economía refleja sus niveles de crecimiento, a través de la evolución del Producto Interno Bruto, PIB, el cual: valora la producción total de los bienes y servicios finales que han sido realizados durante un año en un país. Esta medición, no incluye, a los productos elaborados por las personas del país residentes en el extranjero y sí incorpora los producidos por extranjeros residentes en el país. (Mankinw, 2014)

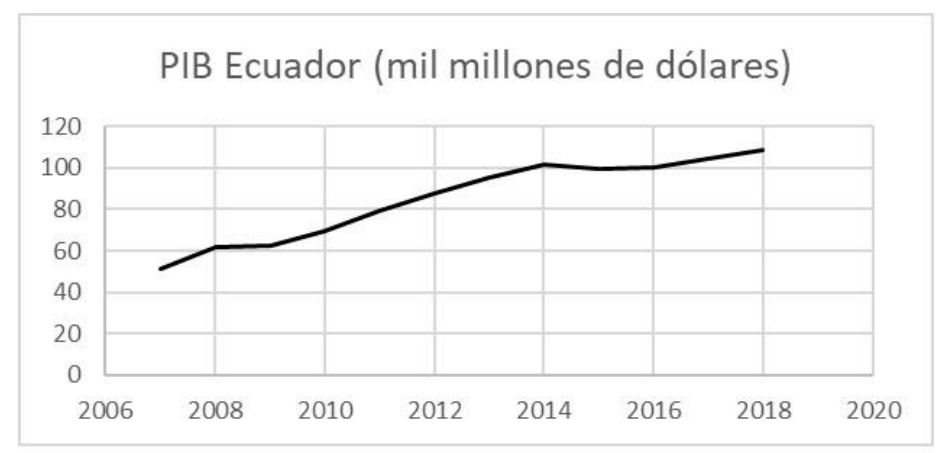

Figura 1: Evolución PIB del Ecuador 2007 - 2018

Fuente: Elaboración autores.

Esta figura muestra la evolución que ha presentado el PIB del Ecuador desde el año 2007, hasta el año 2018, los datos están expresados en moneda local a precios constantes. Se evidencia como el PIB en el Ecuador ha presentado niveles de crecimiento superiores a los obtenidos en décadas anteriores, lo cual se puede explicar por la inversión determinada por los gobiernos para potenciar la producción e inversión. El 2014 y el 2018 han sido los años con mayor alcance del PIB debido a la adecuación de la economía en el contexto internacional. 
La economía ecuatoriana se ha caracterizado por ser dependiente de las exportaciones de petróleo y gracias al boom petrolero ocurrido durante 2007 - 2014 la economía experimento niveles de crecimiento, sin embargo, el manejo no adecuado de las políticas macroeconómicas generaron problemas estructurales, evidentemente existió una escasa inversión privada, por otro lado el sector público crecía pero de manera ineficiente y además no habían medidas adecuadas de estabilización de la economía, razón por la cual el crecimiento económico empezó a bajar hasta el año 2017. En el 2018 el nivel de producción en el país empieza a recuperarse debido a la restricción del gobierno en cuanto a los gastos del sector público y racionalización de la inversión pública, medidas que han aportado a una recuperación leve de la economía. (Banco Mundial, 2018)

A pesar de los niveles de crecimiento que ha experimentado el PIB, esta productividad aún no ha podido llegar a todos los sectores de la población, ya que aún hay sectores de la economía que no pueden satisfacer sus necesidades debido a los índices de pobreza que el país presenta.

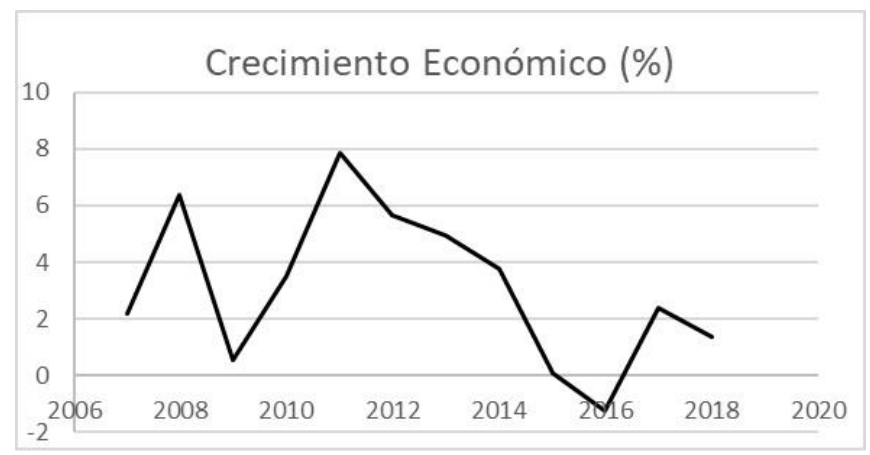

Figura 2: Crecimiento económico del Ecuador 2007 - 2018

Fuente: Elaboración autores

Tasa de crecimiento anual porcentual a precios de mercado en moneda local, a precios constantes. Los agregados están expresados en dólares de los Estados Unidos a precios constantes del año 2010. El Ecuador durante este período ha alcanzado niveles de crecimiento, especialmente en los años 2008 y 2011 ya que fue un período donde un boom petrolero permitió que la economía de alguna manera se fortalezca. Sin embargo, por el desequilibrio de las políticas públicas establecidas, el Ecuador no hay podido potenciar este crecimiento y durante este período ha sido difícil alcanzar un nivel de crecimiento económico sostenible.

La economía ecuatoriana no ha logrado mantener una tasa de crecimiento económico sostenida, ni tampoco los niveles alcanzados reflejan un despunte significativo. Durante el período 2007 - 2014, la economía creció en un promedio del 4.3\%, esto debido a los altos precios del petróleo y el excesivo gasto público que ejercía el gobierno a través de la construcción de nuevas carreteras y edificaciones. Este crecimiento promedio a pesar de la 
bonanza petrolera existente no pudo superar al crecimiento promedio de la década anterior que fue del $4.9 \%$, lo cual evidencia que la economía ecuatoriana se estancó debido al mal manejo de los ingresos generados por la producción. A partir del 2015, hubo un decrecimiento hasta el 2018, el cual en promedio registro un valor del $0.4 \%$, debido a que la economía debía empezar a enfrentar el pago de las deudas generadas por el excesivo gasto público.

\section{Deuda}

La implementación y potenciación de las distintas políticas generadoras de un crecimiento económico en un país dependen de los ingresos que genera un país y del endeudamiento, por tal razón el endeudamiento debe servir para aportar a la mejora de resultados, ya que si esto no sucede significa que no ha existido un planeamiento adecuado de la política económica.

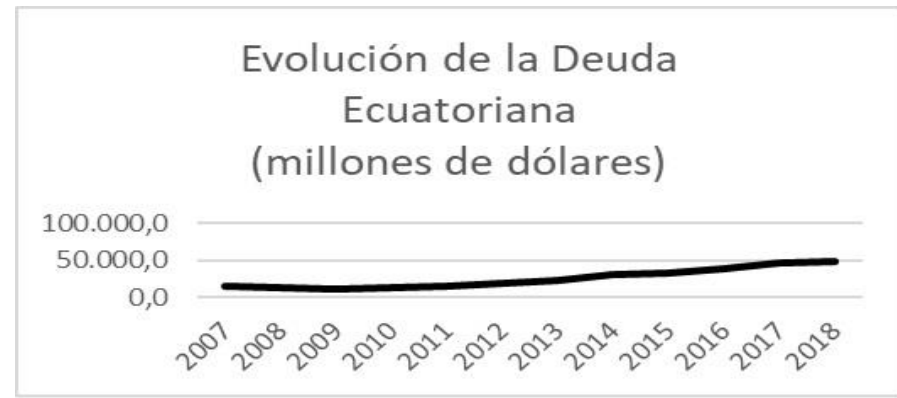

Figura 3: Evolución de la Deuda Pública 2007 - 2018 Fuente: Elaboración

La escasa generación de ingresos que la economía ecuatoriana ha generado durante los últimos años y el excesivo gasto público que los dos últimos gobiernos han mantenido durante su mandato, ha sido el detonante para que la economía ecuatoriana sea dependiente del endeudamiento, el nivel de deuda pública se ha incrementado por la escasa capacidad del sector productivo por generar ingresos suficientes y sostenibles a un largo plazo.

El Ecuador durante su historia económica, ha presentado altos índices de déficit fiscal, el cual se ha financiado tradicionalmente por deuda, a través de la emisión de préstamos $\mathrm{u}$ obligaciones, el Ecuador a nivel público se ha endeudado con entidades del sector Público, IESS y Banco del Estado entre alguna de ellas, a nivel privado mantiene deudas con Organismos Internacionales, Gobiernos a nivel mundial, Bancos Internacionales y con Créditos que se establecen con los proveedores. (Observatorio de la Política Fiscal, 2018)

Si la economía ecuatoriana requiere un endeudamiento para el establecimiento de ajustes macroeconómicos que, aunque sean políticamente costosos permitan de alguna manera una mejora económica, en este sentido el país no puede continuar con la política establecida desde 
el 2014, en la cual se gastaba el dinero ajeno de manera irresponsable, específicamente en gasto corriente. De acuerdo con los datos del Observatorio de la Política Fiscal, la deuda pública externa e interna representa el 51\% del PIB, es decir 55.648 millones de dólares.

\section{Pobreza}

La pobreza es una problemática que afecta de manera significativa a toda la población a nivel mundial, ya que la mismo limita el bienestar de los individuos por el escaso acceso de acceder a programas de salud, educación, seguridad, también ocasiona que la población no cuente con los recursos necesarios para la satisfacción de sus necesidades. La pobreza se ha convertido en un problema que afecta a las condiciones de vida, razón por la cual a muchos países han establecido programas de desarrollo que permitan la creación de nuevas oportunidades de generar condiciones adecuadas de vida. (Programa de las Naciones Unidas para el Desarrollo, 2016)

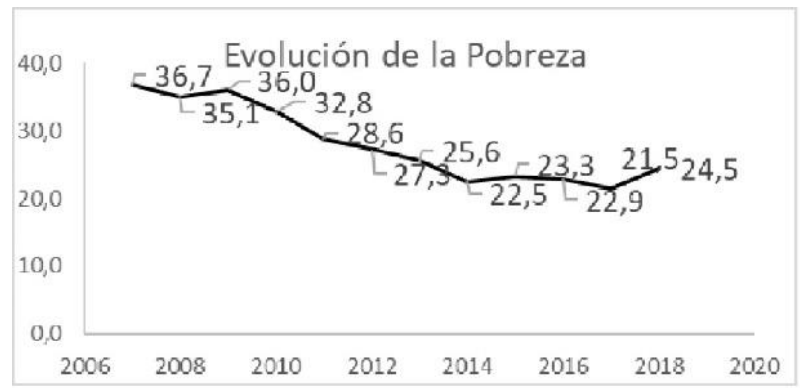

Figura 4: Evolución de los niveles de pobreza 2007 - 2018 Fuente: Elaboración autores

Las políticas económicas establecidas durante el gobierno del Economista Rafael Correa se implementaron con la finalidad de permitir que más personas tengan acceso a los servicios básicos necesarios y así contribuir a una calidad de vida adecuada. Estos niveles de pobreza que se presentan se han medido a través de un método indirecto que mide la pobreza en relación con los ingresos que la población percibe y la capacidad para adquirir los bienes de la canasta básica.

Para analizar los índices de pobreza en el país, es necesario establecer distintas etapas. La primera comprende el período entre 2007-2015, ya que durante este tiempo el gobierno estableció un Comité Institucional para la Erradicación de la Pobreza, el cual buscó crear programas y acciones que permitan la generación de empleo estables y el acceso a los servicios básicos, razón por la cual durante este tiempo hubo una reducción significativa de la pobreza, de 36,7\% al 23,3\%. Al cierre del 2018\% la pobreza en el país se situó en 24,5\%, lo cual si bien es cierto es inferior al índice de pobreza inicial, no es un índice apropiado, ya que aún existen sectores de la población que carecen de recursos económicos y por esta razón 
tienen dificultad para acceder a los servicios de salud, educación y sus oportunidades para encontrar plazas de empleo son limitadas.

\section{Recaudación tributaria}

El instrumento que permite decidir sobre la economía del Ecuador es la política fiscal, la cual centra su accionar en la recaudación de impuestos. Durante el período analizado se efectuaron cambios en el ámbito social, político económico y fiscal. En el ámbito fiscal se establecieron políticas que permitiesen una mayor recaudación tributaria gracias al accionar de las empresas públicas y a los ingresos derivados de la exportación petrolera.

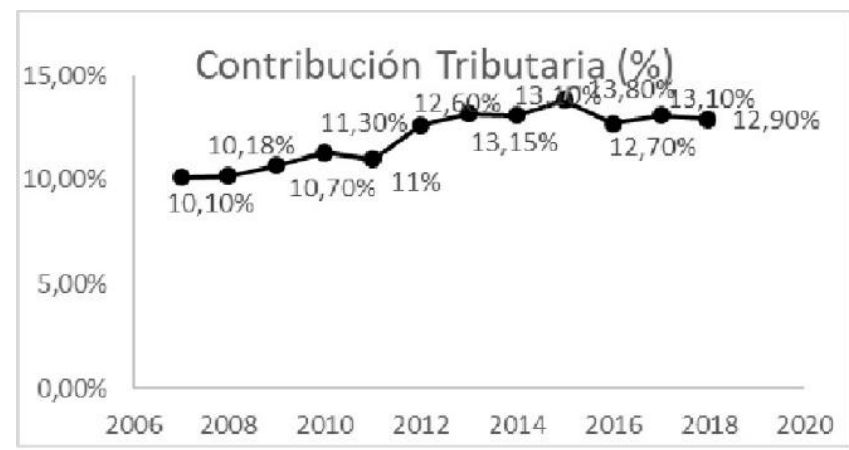

Figura 5: Evolución de la contribución tributaria al PIB 2007 - 2018

Fuente: Elaboración autores

La cifra de recaudación tributaria durante el período de análisis se ha mantenido en un valor no muy variable, esta recaudación está compuesta por el impuesto a la renta, impuesto al valor agregado y otro tipo de impuestos. El modelo económico establecido en el Ecuador se desarrollaba en base a la imposición de una política tributaria creciente, con la finalidad de contar con mayores que permitan corregir el déficit fiscal que el país presenta. (Servicio de Rentas Internas, 2018)

Durante el periodo 2007 - 2014, el país recaudó considerables montos en el tema tributario, ya que se dio prioridad a los impuestos directos y progresivos, con la finalidad de disminuir los altos índices de desigualdad existentes en la población y evitar la alta concentración de la riqueza en pequeños segmentos de la población. A partir del 2015, la recaudación tributaria se ha mantenido en niveles promedio del $13 \%$ de participación en el PIB, lo cual refleja que durante estos años el crecimiento económico del país ha ido de la mano en relación con los tributos recolectados de la población. La política fiscal durante este período de análisis evidencia que los gobiernos se han concentrado en la generación de ingresos como mecanismo para alcanzar los objetivos de la economía. En el 2018, se evidencia una tendencia a la baja de la recaudación, esto debido a que los bajos niveles de crecimiento económico del Ecuador inciden en el volumen de recaudación tributaria. (Urgilés \& Chávez, 2017) 


\section{Conclusiones.}

- A partir de la dolarización, la economía ecuatoriana ha limitado su accionar en materia monetaria, razón por la cual la política fiscal ha tenido que ser diseñada para conseguir un equilibrio fiscal, en el cual haya un reajuste en los gastos desarrollados por el gobierno, pero durante el período analizado esta reducción de gastos se ha centrado en el aspecto social.

- El Ecuador tiene un serio problema de desarrollo a mediano y largo plazo, su producto principal que es el petróleo, no es renovable y se terminará o dejará de ser tan importante en el proceso productivo como lo es hasta el momento. El país debe apostar a pasar de ser un país productor de materia prima, de ser un país dependiente del petróleo a ser un país competitivo en bienes y servicios específicos que permitan ser un país con ventajas absolutas.

- La solución inmediata de corto plazo con relación al déficit fiscal está básicamente en la gestión efectiva de tributos ya que representan cerca del 50\% de los ingresos del Presupuesto General del Estado, razón por la cual el gobierno debe establecer medidas en el ámbito fiscal que permitan la recaudación eficiente de impuestos, ya que de esta manera se garantizará la sostenibilidad de la política fiscal.

\section{Referencias bibliográficas.}

Álvarez, S. (2006). Análisis de la Sostenibilidad de la Política Fiscal y el Efecto del Gasto Público sobre la Economía. 1994-2006. Ecuador.

Asamblea Nacional. (2008). Constitución de la República del Ecuador. http://www.asambleanacional.gov.ec/documentos/constitucion_de_bolsillo.pdf

Banco Mundial. (2018). Ecuador: Panorama Genal. https://www.bancomundial.org/es/country/ecuador/overview

Carrillo, P. (2015). Efectos macroeconómicos de la política fiscal en Ecuador 1993-2009. Analítika, Revista de Análisis Estadístico, 9, 21-52.

Carrillo, P. (2017). El efecto de la política fiscal en expansión y recesión para Ecuador: un modelo MSVAR. Cuadernos de Economía, 36(71), 405-439. doi: 10.15446/ cuad.econ.v36vn72.53570.

Center for Economic and Social Rights, (2017). Política Fiscal para la Igualdad y los Derechos. New York: Fundación Ford, 2017.

CEPAL. (2017). Panorama fiscal de América Latina y el Caribe 2017. La movilización de recursos para el financiamiento del desarrollo sostenible. Naciones Unidas. 
Vol. 4, $\mathrm{N}^{\circ} 1$, p. 81-94, enero - marzo, 2020

Erráez, J. (2014). Sistema de indicadores del ciclo de crecimiento económico. Banco Central del Ecuador

Mankiw, N. (2014). Macroeconomía: versión para América Latina. ( $8^{\mathrm{a}}$ ed.). Barcelona, España: Cengage Learning.

Observatorio de la Política Fiscal. (2018). Transparencia Fiscal. https://www.observatoriofiscal.org/publicaciones/transparencia-fiscal.html

Otálora C. (2009). Economía Fiscal. Bolivia. Plurales editores Programa de las Naciones Unidas para el Desarrollo. (2016). Reducción de la pobreza.

Ecuador. http://www.ec.undp.org/content/ecuador/es/home/ourwork/povertyreduction/ove rview.html

Rozas, P. (2010). América Latina: problemas y desafíos del financiamiento de la infraestructura. Revista de la CEPAL, 101, 59-83.

Samuelson, P. y Nordhaus, W. (2014). Introducción a la Macroeconomía. 18ª ed. Madrid: McGraw-Hill.

Servicio de Rentas Internas. (2018). Proforma presupuestaria 2018. Quito - Ecuador. https://sri.gob.ec

Urgiles, G. \& Chávez, K. (2017). Evolución de la recaudación tributaria y gasto público en el Ecuador durante la última década. Revista Visión Empresarial No 7; pp: 139 - 155. Quito,

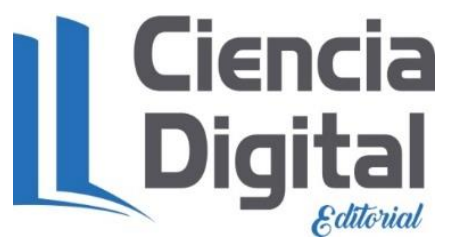




\section{PARA CITAR EL ARTÍCULO INDEXADO.}

Chamba Bastidas, L. A., Morales Noriega, A. M., Vásconez Vásconez, H. D., \& Cabrera Bravo, D. A. (2020). Análisis de la política fiscal descrita en la constitución vigente del Ecuador. Visionario Digital, 4(1), 81-94. https://doi.org/10.33262/visionariodigital.v4i1.1106

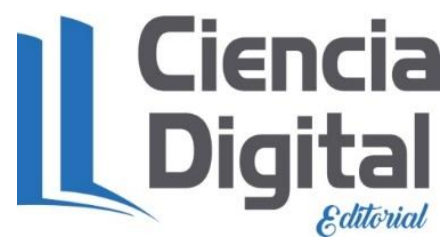

El artículo que se publica es de exclusiva responsabilidad de los autores y no necesariamente reflejan el pensamiento de la Revista Visionario Digital.

El artículo queda en propiedad de la revista y, por tanto, su publicación parcial y/o total en otro medio tiene que ser autorizado por el director de la Revista Visionario Digital.
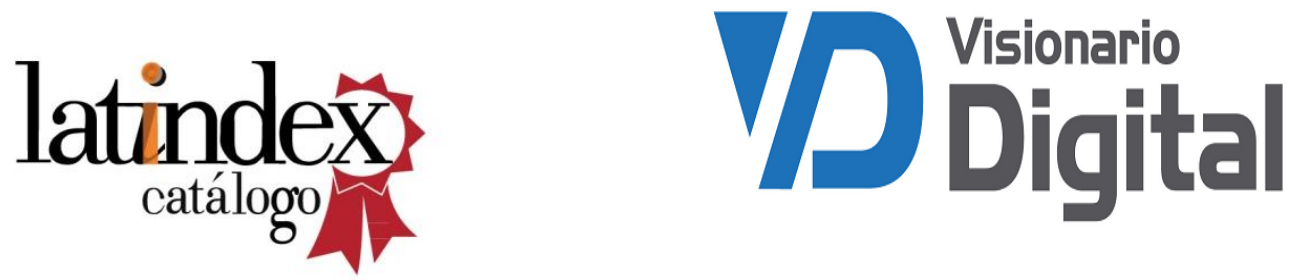